\title{
A randomized crossover clinical study showing that methylphenidate-SODAS improves attention-deficit/hyperactivity disorder symptoms in adolescents with substance use disorder
}

\author{
C.M. Szobot ${ }^{1,2,3}$, L.A. Rohde 2 , B. Katz ${ }^{2}$, P. Ruaro ${ }^{1,3}$, T. Schaefer ${ }^{1,3}$, M. Walcher ${ }^{1,3}$, \\ O. Bukstein ${ }^{4}$ and F. Pechansky ${ }^{1}$
}

${ }^{1}$ Centro de Pesquisas em Álcool e Drogas, ${ }^{2}$ Programa de Déficit de Atenção/Hiperatividade, Hospital de Clínicas de Porto Alegre, Universidade Federal do Rio Grande do Sul, Porto Alegre, RS, Brasil ${ }^{3}$ Curso de Medicina, Universidade Luterana do Brasil, Canoas, RS, Brasil ${ }^{4}$ Western Psychiatric Institute and Clinic, University of Pittsburgh School of Medicine, Pittsburgh, PA, USA

Correspondence to: C.M. Szobot, Centro de Pesquisa em Álcool e Drogas, Hospital de Clínicas de Porto Alegre, Universidade Federal do Rio Grande do Sul, Rua Ramiro Barcelos, 2350, 90035-003 Porto Alegre, RS, Brasil

Fax: +55-51-3330-5813. E-mail: cmszobot@terra.com.br

Our objective was to evaluate the effectiveness of a long-acting formulation of methylphenidate (MPH-SODAS) on attentiondeficit/hyperactivity disorder (ADHD) symptoms in an outpatient sample of adolescents with ADHD and substance use disorders (SUD). Secondary goals were to evaluate the tolerability and impact on drug use of MPH-SODAS. This was a 6-week, singleblind, placebo-controlled crossover study assessing efficacy of escalated doses of MPH-SODAS on ADHD symptoms in 16 adolescents with ADHD/SUD. Participants were randomly allocated to either group A (weeks 1-3 on MPH-SODAS, weeks 4-6 on placebo) or group B (reverse order). The primary outcome measures were the Swanson, Nolan and Pelham Scale, version IV (SNAP-IV) and the Clinical Global Impression Scale (CGI). We also evaluated the adverse effects of MPH-SODAS using the Barkley Side Effect Rating Scale and subject reports of drug use during the study. The sample consisted of marijuana ( $\mathrm{N}=16$; $100 \%)$ and cocaine users $(N=7 ; 43.8 \%)$. Subjects had a significantly greater reduction in SNAP-IV and CGI scores (P < 0.001 for all analyses) during MPH-SODAS treatment compared to placebo. No significant effects for period or sequence were found in analyses with the SNAP-IV and CGI scales. There was no significant effect on drug use. MPH-SODAS was well tolerated but was associated with more severe appetite reduction than placebo $(P<0.001)$. MPH-SODAS was more effective than placebo in reducing ADHD symptoms in a non-abstinent outpatient sample of adolescents with comorbid SUD. Randomized clinical trials, with larger samples and SUD intervention, are recommended.

Key words: Attention deficit/hyperactivity disorder; Methylphenidate; Substance use disorder; Treatment; Adolescents

Research partially supported by CNPq (No. 307780/2004-0) and Hospital de Clínicas de Porto Alegre (financial support for placebo and subject transportation). Toxilab ${ }^{\circledR}$ provided urinary assays. MPH-SODAS was provided by Novartis at no cost and without restrictions. C.M. Szobot is on the speakers' bureau of Janssen-Cilag and had travel support by Novartis. The ADHD outpatient program receives research support from the following pharmaceutical companies: Bristol-Myers Squibb, Eli-Lilly, Janssen-Cilag, and Novartis. L.A. Rohde is on the speakers' bureau or is a consultant for the same companies and is on the advisory board for Eli-Lilly. F. Pechansky's center receives funding for research projects from NIDA, NIAID, and the Brazilian Anti-Drug Secretaria. O. Bukstein is affiliated with the following: Shire Pharmaceuticals - consultant, research support, speaker's bureau; McNeil pediatrics - speaker's bureau, consultant; Eli-Lilly - research support; Novartis - speaker's bureau. 


\section{Introduction}

Adolescent substance use disorder is a major mental health concern in different cultures $(1,2)$. Marijuana is the most abused illicit drug worldwide, with an annual prevalence of $2.5 \%$ and affecting mostly young populations (3). The use of cocaine is also increasing during adolescence, particularly in developing countries (2). The worldwide prevalence of attention deficit/hyperactivity disorder (ADHD) in adolescents was recently estimated by our group in a meta-analysis of the literature $(2.74 ; 95 \% \mathrm{Cl}=2.04-3.45)$ (4). These figures are even higher in non-referred samples from developing countries $(5,6)$. The prevalence rates and associated impairments also qualify ADHD as a major public health concern (7).

ADHD is highly prevalent among adolescents with substance use disorders (SUD) $(8,9)$. The comorbidity is clinically relevant, since ADHD is associated with both earlier and more frequent alcohol relapses (10) and a lower likelihood of cannabis treatment completion (11) in adolescents. Several evidence-based guidelines have suggested that stimulants such as methylphenidate (MPH) should be the first option for treatment of ADHD (see, for instance, Pliszka et al., 12). However, ADHD treatment studies typically exclude individuals with drug use/misuse or SUD. Given that most abused drugs act on the dopaminergic system (13), as does MPH (14), pharmacological studies of subjects with ADHD/SUD are crucial.

Few open trials with bupropion $(15,16)$ and one randomized clinical trial with pemoline (17) have addressed adolescents with ADHD/SUD. All of them reported significant treatment effects in reducing ADHD symptoms. Interestingly, first line medications for ADHD were recently evaluated in adults with the comorbidity, with a positive result regarding ADHD symptoms (18), and most of the few available randomized clinical trials did not find a superior effect of $\mathrm{MPH}$ over placebo $(19,20)$, or over placebo and bupropion (21) on ADHD symptoms. These findings may not necessarily be translated to adolescents, since there are reports of different MPH (22) and drug responses $(23,24)$ between adults and adolescents. Thus, the main goal of the present study was to evaluate the effectiveness of a long-acting formulation of $\mathrm{MPH}(\mathrm{MPH}-$ SODAS) in reducing ADHD symptoms in adolescents with ADHD/SUD compared to placebo. Secondarily, we evaluated drug consumption and MPH-SODAS tolerability.

\section{Subjects and Methods}

\section{Study design and participants}

This was a 6-week, randomized, single-blind, placebocontrolled crossover trial assessing the effects of MPH-
SODAS on ADHD symptoms in 16 adolescent male outpatients with both ADHD and SUD. Subjects were randomly allocated to two groups. Group A received MPH-SODAS while group $B$ received placebo during the first 3 weeks. In weeks 4-6, group A received placebo and group B received MPH-SODAS. The study was conducted between November and December 2005 in the city of Porto Alegre (capital of the Brazilian Southernmost State, Rio Grande do Sul). Figure 1 summarizes study design and procedures.

The project was approved by the Institutional Review Board (IRB) of Hospital de Clínicas de Porto Alegre (approved as an IRB by the Office for Human Research Protections, United States of America, IRB 00000921). Written informed consent was obtained from all participants and their parents. Subjects were recruited from a previous community case-control study assessing adolescents with ADHD/SUD (25). Of the 21 eligible subjects, 11 accepted to be enrolled in the study. We also recruited more subjects by advertisements in local newspapers and radio broadcasts; from this source, we screened 15 adolescents. Eleven youths had ADHD/SUD; however, 3 subjects met exclusion criteria (see below) and 3 refused to participate. The other 5 adolescents were included in the study, thus comprising our total sample size of 16 subjects. Inclusion criteria were age between 15 and 21 years, male gender, current diagnosis of abuse of or dependence on marijuana or cocaine, current diagnosis of ADHD, and stimulant-naive subjects. Exclusion criteria were the lack of a responsible adult to inform about possible childhood psychopathology or to take responsibility for the medication, the need for inpatient treatment for drug abuse or psychiatric comorbidities, and the presence of a primary psychiatric condition that required immediate outpatient treatment (like moderate/severe depression). SUD treatment was not provided. Drug and alcohol abstinence was not required for study eligibility.

\section{Study medication procedures}

A pharmacist packaged MPH-SODAS and matching placebo in capsules, so that MPH-SODAS and placebo could not be visually differentiated. One of the investigators (LAR) randomized the 16 subjects into groups $A$ or $B$, and prepared weekly blisters of medications for each participant. MPH-SODAS is suitable for crossover designs since its clinical response is limited to no more than 8-9 h (26), preventing carryover effects.

The medication was given to the subjects' mothers, and was taken once a day (in the morning) by oral administration. Study compliance was assessed by self-report, mother's report and pill counting. Medication doses were 0.3, 0.7 and $1.2 \mathrm{mg} \cdot \mathrm{kg}^{-1} \cdot \mathrm{day}^{-1}$, for weeks 1,2 and 3 for 
group A, and weeks 4, 5 and 6 for group B. In Figure 1, a flowchart of study design and procedures is shown.

\section{Diagnostic procedures}

The diagnoses of ADHD and comorbid mental disorders were confirmed by semi-structured interviews (Schedule for Affective Disorders and Schizophrenia for SchoolAge Children, Epidemiological Version, K-SADS-E) with the parents, and clinical interviews with the adolescent and the parents conducted by a child psychiatrist (CMS). Detailed description of the diagnostic process in our ADHD clinic can be found elsewhere (27). The diagnoses of SUD relied on the drug section of the Mini International Neuropsychiatry Interview (MINI), Brazilian version (28), which generates diagnoses of abuse or dependence according to Diagnostic and Statistical Manual of Mental Disorders (DSM-IV) criteria. All participants had drug use confirmed by urinary tests (cannabis and/or cocaine). Other measures were: a) the Clinical Global Assessment Scale (CGAS) (29); b) the Brazilian Association of Market Research Form (30) for evaluation of socioeconomic status (SES); c) Block Design and Vocabulary subtests of the Wechsler Intelligence Scale, third edition (WISC-III) (31), and the Wechsler Adult Intelligence Scale (WAIS) (32) for estimation of IQ.

\section{Outcome measures: efficacy assessments}

The primary outcome measures (efficacy assessment) were the Swanson, Nolan and Pelham Scale, version IV (SNAP-IV) (33) and the Clinical Global Impression (CGI) Scale (34) (Severity, Clinical Improvement and Efficacy scores). The SNAP-IV is a 26 -item scale based on DSM-IV ADHD and oppositional defiant disorder symptoms. Items are scored on a 4-point scale [from 0, never (meaning a symptom that never happens), to 3 , very often (meaning a symptom that happens very frequently)]. In the present study, the SNAP-IV evaluation was based on the mother's report. The CGI scale is an investigator-rated scale which consists of 3 subscales: severity, clinical improvement and efficacy. The CGI severity consists of a 7-item scale (from 0 , not evaluated, to 7 , extremely ill). The CGI clinical improvement is also a 7-item scale (from 0, not evaluated, to 7, much worse) and the CGI efficacy combines adverse events with clinical improvement (from 1, no adverse effect/notable clinical improvement, to 16 , adverse effect is higher than beneficial effects/no clinical improvement/clinically worse).

With regard to secondary outcome measures, drug use was evaluated by the number of days with drug use (weekly), the number of smoked cannabis cigarettes (weekly) and

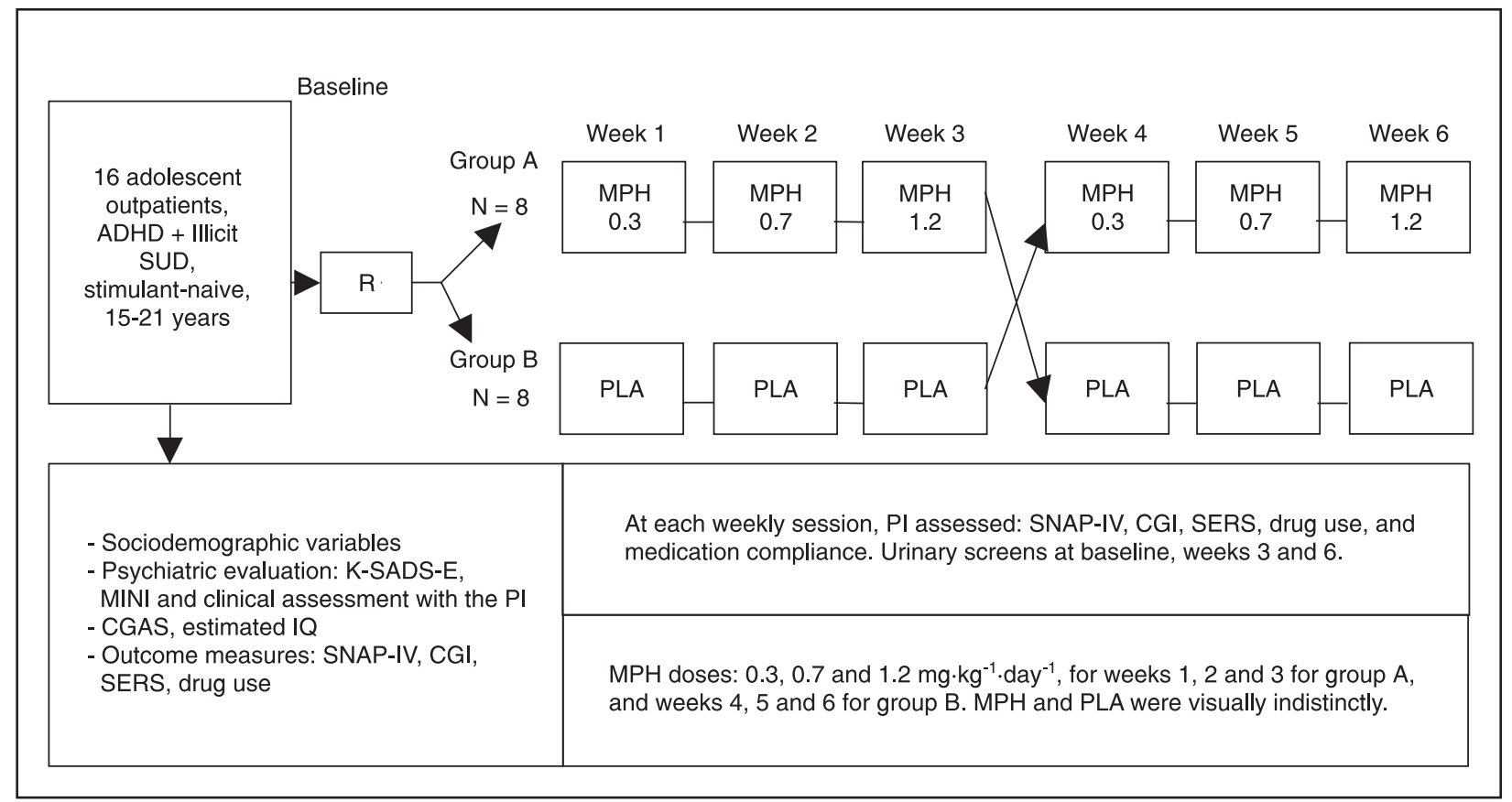

Figure 1. Flowchart of study design and procedures. $A D H D=$ attention deficit/hyperactivity disorder; SUD = substance use disorder; $\mathrm{R}=$ randomization; K-SADS-E = Schedule for Affective Disorders and Schizophrenia for School - Age Children, Epidemiological Version; MINI = Mini International Neuropsychiatry Interview; PI = principal investigator; CGAS = Clinical Global Assessment Scale; IQ = Intelligence quotient; SNAP-IV = Swanson, Nolan, and Pelham Scale, version IV; CGI = Clinical Global Impression Scale; SERS = Barkley Side Effect Rating Scale; MPH = methylphenidate SODAS; PLA = placebo. 
by a urinary test for cannabis and cocaine (weeks 3 and 6). Tolerability consisted of the total score of the Barkley Side Effect Rating Scale (SERS) (35), and of its insomnia, headache and appetite subitems.

\section{Data analysis}

Analyses of primary and secondary outcome measures were performed using a mixed-effect model (MEM) approach which provides a flexible framework for the analysis of repeated measures while accounting for missing data (i.e., lost to follow-up) (36). We used treatment, period, sequence, and dose-within-treatment as fixed variables, baseline measures as covariates (excepts for CGI clinical improvement and efficacy, which does not have baseline values) and subjects as a random variable. For each analysis, the best covariance structure fitting the data was selected using Akaike's information criterion (AIC) (37). A significance level of $5 \%$ was set for all analyses.

\section{Results}

Two participants, both from group A, dropped out of the study. One of them started an intense use of inhalants, requiring hospitalization, by the end of the third week. The other one felt worse (more restless) during the fourth week, and dropped out of the study (dropout rate $=12.5 \%$ ).

\section{Sample characteristics}

Most of the subjects had a cannabis SUD diagnosis (approximately 3 years of regular use) and 7 (43\%) also had cocaine abuse or dependence (current or past). With regard to current diagnosis of cocaine, one subject from group A had abuse and one had dependence. In group $B$, 2 adolescents had current abuse and one had dependence. In addition, 5 (31.3\%) adolescents had an alcohol use disorder. Subjects' mean SNAP-IV and CGAS baseline scores were $50.63(S D=13.76)$ and $42.19(S D=9.12)$,

Table 1. Sociodemographic and clinical characteristics of the participants according to study randomization.

\begin{tabular}{|c|c|c|c|c|}
\hline \multirow[t]{2}{*}{ Characteristic } & \multicolumn{2}{|c|}{ Group A $(N=8)$} & \multicolumn{2}{|c|}{ Group B $(\mathrm{N}=8)$} \\
\hline & Mean (SD) & N (\%) & Mean (SD) & N (\%) \\
\hline Age (years) & $17.50(2.33)$ & & $17.38(2.2)$ & \\
\hline Ethnicity (European-Brazilian) & & $3(37.5 \%)$ & & $7(87.5 \%)$ \\
\hline \multicolumn{5}{|l|}{ Socioeconomic level } \\
\hline$A+B+C$ & & $4(50 \%)$ & & $7(87.5 \%)$ \\
\hline$D+E$ & & $4(50 \%)$ & & $1(12.5 \%)$ \\
\hline Divorced parents & & $3(37.5 \%)$ & & $4(50 \%)$ \\
\hline School (mean grade) & $7.13(2.23)$ & & $7.75(1.67)$ & \\
\hline Estimated IQ & $79.43(16.66)$ & & $84.75(21.16)$ & \\
\hline \multicolumn{5}{|l|}{ SNAP-IV } \\
\hline Total score & $50.38(18.05)$ & & $50.38(8.93)$ & \\
\hline Inattentive score & $18.13(7.26)$ & & $18.63(5.50)$ & \\
\hline Hyperactive score & $18.75(7.38)$ & & $19.25(5.26)$ & \\
\hline ODD score & $13.38(6.63)$ & & $13.0(5.40)$ & \\
\hline \multicolumn{5}{|l|}{ Further lifetime DSM-IV axis diagnosis } \\
\hline Conduct disorder & & $8(100 \%)$ & & $6(75 \%)$ \\
\hline Oppositional defiant disorder & & $2(25 \%)$ & & $3(37.5 \%)$ \\
\hline Depression & & $1(12.5 \%)$ & & $2(25 \%)$ \\
\hline CGI severity & $5.88(0.84)$ & & $5.50(0.55)$ & \\
\hline Clinical Global Assessment Scale & $44.38(12.08)$ & & $40.0(4.63)$ & \\
\hline \multicolumn{5}{|l|}{ SUD } \\
\hline Marijuana & & $8(100 \%)$ & & $7(87.5 \%)$ \\
\hline Cocaine & & $4(50 \%)$ & & $3(37.5 \%)$ \\
\hline Days of cannabis use, last month & $30(0.0)$ & & $28.57(3.78)$ & \\
\hline Number of cannabis cigarettes per day & $3(0.76)$ & & $2.71(0.95)$ & \\
\hline
\end{tabular}

Data are reported as mean with SD in parentheses and as number of subjects with percent in parentheses. $I Q=$ intelligence quotient; SNAP-IV = Swanson, Nolan, and Pelham Scale, version IV; ODD = oppositional defiant disorder; DSM-IV = Diagnostic and Statistical Manual of Mental Disorders; CGI = Clinical Global Impression Scale; SUD = substance use disorder. Group A: methylphenidateSODAS (MPH-SODAS) during weeks 1, 2, and 3, and placebo during weeks 4, 5, and 6. Group B: placebo during weeks 1, 2, and 3, and MPH-SODAS during weeks 4, 5, and 6 . There were no significant differences between groups A and B (for continuous variables, $t$-test for independent sample; for dichotomous variables, chi-square test). 
respectively. The most common type of ADHD was the combined one $(\mathrm{N}=12,75 \%)$, followed by the inattentive ( $\mathrm{N}$ $=3,18.75 \%)$ and the hyperactive/impulsive type $(\mathrm{N}=1)$. In addition to ADHD and SUD, 14 subjects (87.5\%) had an additional (current or lifetime) diagnosis of conduct disorder, 5 (31.25\%) of oppositional defiant disorder, 3 (18.75\%) of major depression, and one of separation anxiety (this last subject belonged to group B). Socio-demographical characteristics, estimated IQ, psychiatric disorders, ADHD severity, and drug use of the sample according to group randomization can be found in Table 1. There were no significant differences in any of these variables according to sequence (comparison between subjects receiving MPH or placebo in the first period; $P>0.05$ ).

\section{Primary outcome measures: SNAP-IV and CGI}

The MEM analyses revealed highly significant MPHSODAS treatment effect on ADHD symptoms and on subject functioning compared to placebo, according to both SNAP-IV and CGI scores ( $P \leq 0.001$ for all analyses). There was no significant sequence or period effect, although we detected a trend for a sequence effect in both inattentive and hyperactivity/impulsivity dimensions of the SNAP-IV scale ( $P=0.06$ for both analyses). As expected, baseline SNAP-IV and CGI severity scores were significantly associated with response to treatment $(P \leq 0.001$ for all analyses). There were also significant dose-withintreatment effects - only for CGI severity and CGI clinical improvement scores $(P \leq 0.001$ for both analyses; see Table 2 and Figure 2).

With regard to the number of days with drug use, there

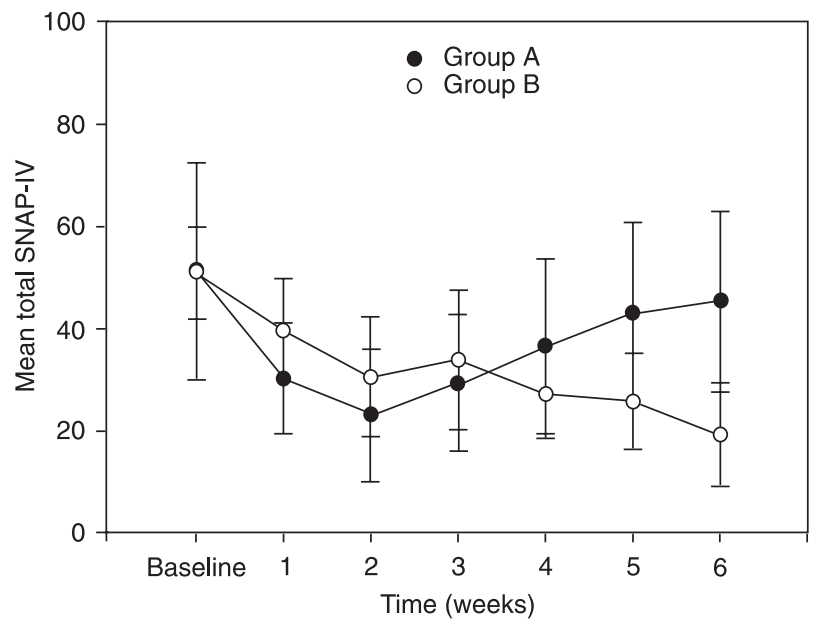

were no significant treatment $(P=0.10)$, period $(P=0.62)$ or order effects $(P=0.37)$, but there were significant effects for baseline score $[F(1,14)=54211.33, P \leq 0.001]$ and dose-within-treatment $[F(1,14)=3.22, P=0.04]$. When

Table 2. Mixed-effect model analyses of the effects of methylphenidate-SODAS vs placebo on SNAP-IV and CGI scores in adolescents with both attention deficit/hyperactivity disorder and illicit substance use disorder $(\mathrm{N}=16)$.

Treatment Period Sequence Dose-withintreatment

\begin{tabular}{llrrr}
\hline SNAP totala $^{a}$ & & & & \\
F $(1,14)$ & 42.92 & 20.77 & 16.08 & 29.57 \\
P value & $\leq 0.001$ & 0.33 & 0.11 & 0.16 \\
SNAP inattentive ${ }^{\mathrm{b}}$ & & & & \\
$\quad$ F $(1,14)$ & 14.31 & 12.40 & 12.43 & 11.41 \\
$\quad$ P value & 0.001 & 0.99 & 0.06 & 0.34 \\
SNAP hyperactivityc & & & & \\
F $(1,14)$ & 28.75 & 23.02 & 13.35 & 59.80 \\
P value & $\leq 0.001$ & 0.77 & 0.06 & 0.64 \\
CGI severity & & & & \\
F $(1,14)$ & 13.67 & 13.54 & 13.91 & 13.55 \\
P value & $\leq 0.001$ & 0.51 & 0.19 & 0.001 \\
CGI clinical improvement & & & & \\
F $(1,14)$ & 25.31 & 18.97 & 21.33 & 32.28 \\
P value & $\leq 0.001$ & 0.6 & 0.26 & $\leq 0.001$ \\
CGl efficacye & & & & \\
F $(1,14)$ & 72.58 & 72.58 & 14.11 & 71.21 \\
P value & $\leq 0.001$ & 0.65 & 0.21 & 0.24 \\
\hline
\end{tabular}

Significant values are highlighted in bold. Best Covariance Structure: aToeplitz; bUnstructured; ' $A R M A(1,1)$; dFirst-order-ante-dependence; eCompound symmetry. SNAP-IV = Swanson, Nolan, and Pelham Scale, version IV; CGI = Clinical Global Impression Scale.

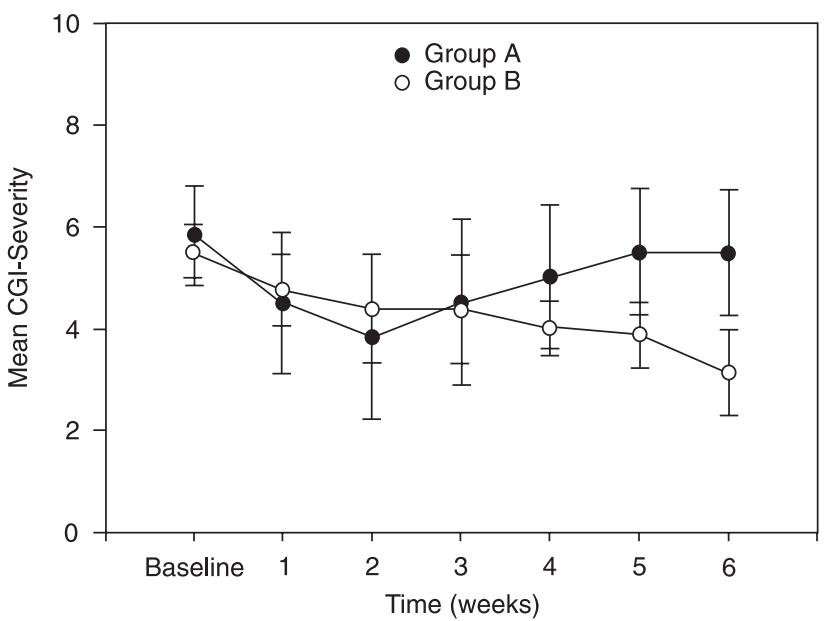

Figure 2. SNAP-IV and CGI-Severity scores during the protocol for MPH-SODAS and placebo groups in adolescents with ADHD and SUD $(N=16)$. SNAP-IV = Swanson, Nolan, and Pelham Scale, version IV; CGI = Clinical Global Impression Scale; ADHD = attention deficit/hyperactivity disorder; SUD = substance use disorder. Group A: methylphenidate SODAS (MPH-SODAS) during weeks 1, 2, 3, and placebo during weeks 4, 5, and 6. Group B: placebo during weeks 1, 2, 3, and MPH-SODAS during weeks 4, 5 , and 6 . 
using MPH-SODAS, subjects presented a slight decrease in the number of days with drug use while doses of medication were increased (5.94 days, SD $=2.02$ at $0.3 \mathrm{mg} \cdot \mathrm{kg}^{-1}$. day $^{-1} ; 5.87$ days, SD $=2.03$ at $0.7 \mathrm{mg} \cdot \mathrm{kg}^{-1} \cdot$ day $^{-1}$, and 5.56 days, $S D=2.03$ at $1.2 \mathrm{mg} \cdot \mathrm{kg}^{-1} \cdot \mathrm{day}^{-1}$ ). However, while on placebo, there was no change in drug use (6.13 days, SD $=2.29$ at $0.3 \mathrm{mg} \cdot \mathrm{kg}^{-1} \cdot$ day $^{-1} ; 5.87$ days, $\mathrm{SD}=2.07$ at 0.7 $\mathrm{mg} \cdot \mathrm{kg}^{-1} \cdot \mathrm{day}^{-1}$, and 6.0 days, SD $=2.10$ at $1.2 \mathrm{mg} \cdot \mathrm{kg}^{-1}$. day $\left.^{-1}\right)$. As for the number of marijuana cigarettes per day, there were no significant treatment, period, order, baseline score, or dose-within-treatment effects $(P \geq 0.2$ in all analyses). There was also no significant change in the status of urine tests for either cannabis or cocaine throughout the study ( $P=1$ in all analyses). We had no report of $\mathrm{MPH}$ misuse.

Medication was well tolerated and we did not detect any significant treatment $(P=0.90)$, order $(P=0.51)$ or dose-within-treatment $(P=0.90)$ effects on SERS total score, although significant period $[F(1,14)=7.52, P=0.02]$ and baseline score effects $[F(1,14)=115.69, P \leq 0.001]$ were found. Treatment with MPH-SODAS significantly reduced appetite $[F(1,14)=15.70, P \leq 0.001]$, with no period effect $(P=0.38)$, but a significant order effect $[F(1,14)=$ $5.65, P=0.03$ ] was found. There were trends to baseline score $(P=0.06)$ and dose-within-treatment $(P=0.07)$ effects. No treatment effect was found for insomnia or headache (analyses available upon request).

\section{Discussion}

In the present study, MPH-SODAS was significantly superior to placebo in reducing ADHD symptoms and improving global functioning for all main outcome measures (SNAP-IV and CGI scores). There was no treatment effect on illicit SUD and MPH-SODAS was well tolerated, despite causing more appetite reduction than placebo.

Our results showing a treatment effect for $\mathrm{MPH}$ on ADHD symptoms in adolescents with illicit SUD are in contrast with some randomized clinical trials with stimulants in adults (19-21). A combination of factors, such as MPH dosage, type of MPH delivery system, type of abused drug, and brain development stage might explain these contradictory findings. In our study, for example, we administered higher $\mathrm{MPH}$ doses than those utilized in the study by Carpentier et al. (19), whose participants used up to $0.6 \mathrm{mg} \cdot \mathrm{kg}^{-1} \cdot \mathrm{day}^{-1} \mathrm{MPH}$. Besides, our sample consisted mostly of marijuana plus cocaine users, in contrast to cocaine $(19,20)$ and cocaine/heroin $(21)$ users in adults' protocols. Moreover, neurobiological differences between adults and adolescents may influence MPH response. It is well established that SUD progression affects the reward system (38). Since MPH acts on reward system (39), the length of time of drug use might affect brain response to $\mathrm{MPH}$. Also, animal studies documented different brain and behavioral responses to cocaine among adolescent vs adult rats (24). Last, the adolescent brain is still in development (40), which might favor recruitment of some brain pathways involved in the effects of MPH. In this respect, our data are in agreement with the only adolescent randomized clinical trial available, which reported the effect of pemoline treatment on ADHD symptoms (17).

There was no significant treatment effect on drug use. Even though some studies have shown that ADHD severity might worsen SUD prognosis $(10,11)$, it is possible to speculate that ADHD improvement within a short period of time ( 3 weeks) is not enough to decrease drug use in adolescents with chronic use. In this respect, Riggs et al. (17) also found no drug use improvement despite ADHD improvement. However, Solhkhah et al. (16) reported a decrease in drug use in 14 adolescents (6-month bupropion open trial), concomitant with a drug use intervention. It is important to emphasize that our protocol lasted for a shorter period of time, and we had no specific intervention for SUD. Even so, we detected a similar pattern of drug use during the protocol for MPH-SODAS and placebo (no increase in drug use for either group). Further randomized clinical trials with longer periods, incorporating combined SUD intervention and adjusting results for age at onset of SUD, will help clarify the impact of ADHD treatment on SUD. Regarding MPH-SODAS tolerability, we had no serious adverse event.

Our study should be considered within the context of some of its limitations. First, it was a single-blind protocol. Second, SNAP-IV inattentive and hyperactivity/impulsivity scores tended to show a sequence effect $(P=0.06)$. Pharmacological properties of MPH (short period of time required to produce clinically significant effects) might facilitate such pattern in a crossover study. Thus, youths who started on MPH-SODAS were less prone to placebo effects than participants who started on placebo (group B), since the former might quickly recognize effects of stimulants in their ADHD symptoms, being more prone to present a lower clinical response when switched to placebo. Also, our sample size was small, limiting the power for some analyses (e.g., assessment of interactions) and findings cannot be generalized to adolescents with a different pattern of drug use. Furthermore, our trial lasted 6 weeks, a time that might not be sufficient to appreciate improvement (decrease) in drug use. Also, the presence of other psychiatric comorbidities in the sample (Table 1) might have some impact on treatment effects, although the comorbidity profile did not differ between groups. Despite 
these caveats, our study has some specific strengths: this was the first study to evaluate stimulant effect in a nonabstinent outpatient sample of adolescents with ADHD/ SUD in a placebo-controlled trial enrolling only treatment naive subjects. Moreover, the sample consisted mostly of marijuana users and we had a high retention rate (87\%).

\section{References}

1. Bukstein OG, Bernet W, Arnold V, Beitchman J, Shaw J, Benson RS, et al. Practice parameter for the assessment and treatment of children and adolescents with substance use disorders. J Am Acad Child Adolesc Psychiatry 2005; 44: 609-621.

2. Galduroz JC, Noto AR, Nappo SA, Carlini EA. Household survey on drug abuse in Brazil: study involving the 107 major cities of the country - 2001. Addict Behav 2005; 30: 545-556.

3. WHO. World Health Organization. http://www.who.int/ substance_abuse/facts/cannabis/en/index.html. Accessed January 8, 2006.

4. Polanczyk G, de Lima MS, Horta BL, Biederman J, Rohde LA. The worldwide prevalence of ADHD: a systematic review and metaregression analysis. Am J Psychiatry 2007; 164: 942-948.

5. Rohde LA, Biederman J, Busnello EA, Zimmermann $\mathrm{H}$, Schmitz M, Martins S, et al. ADHD in a school sample of Brazilian adolescents: a study of prevalence, comorbid conditions, and impairments. J Am Acad Child Adolesc Psychiatry 1999; 38: 716-722.

6. Rohde LA. ADHD in Brazil: the DSM-IV criteria in a culturally different population. J Am Acad Child Adolesc Psychiatry 2002; 41: 1131-1133.

7. Lesesne C, Abramowitz A, Perou R, Brann E. Attention deficit/hyperactivity disorder: A public health research agenda. http://www.cdc.gov/ncbddd/adhd/dadphra.htm. Accessed January 8, 2006.

8. Horner BR, Scheibe KE. Prevalence and implications of attention-deficit hyperactivity disorder among adolescents in treatment for substance abuse. J Am Acad Child Adolesc Psychiatry 1997; 36: 30-36.

9. Kuperman S, Schlosser SS, Kramer JR, Bucholz K, Hesselbrock V, Reich $T$, et al. Developmental sequence from disruptive behavior diagnosis to adolescent alcohol dependence. Am J Psychiatry 2001; 158: 2022-2026.

10. Ercan ES, Coskunol H, Varan A, Toksoz K. Childhood attention deficit/hyperactivity disorder and alcohol dependence: a 1-year follow-up. Alcohol Alcohol 2003; 38: 352356.

11. White AM, Jordan JD, Schroeder KM, Acheson SK, Georgi $B D$, Sauls $G$, et al. Predictors of relapse during treatment and treatment completion among marijuana-dependent adolescents in an intensive outpatient substance abuse program. Subst Abus 2004; 25: 53-59.

12. Pliszka SR, Crismon ML, Hughes CW, Corners CK, Emslie GJ, Jensen PS, et al. The Texas Children's Medication Algorithm Project: revision of the algorithm for pharmacotherapy of attention-deficit/hyperactivity disorder. J Am Acad Child Adolesc Psychiatry 2006; 45: 642-657.
To the best of our knowledge, this is the first study suggesting the effectiveness of stimulants on ADHD symptoms in adolescents with ADHD/SUD. However, randomized clinical trials with larger samples, drug treatment intervention and longer follow-ups, are recommended.
13. Volkow ND, Fowler JS, Wang GJ. The addicted human brain viewed in the light of imaging studies: brain circuits and treatment strategies. Neuropharmacology 2004; 47 (Suppl 1): 3-13.

14. Volkow ND, Wang GJ, Fowler JS, Ding YS. Imaging the effects of methylphenidate on brain dopamine: new model on its therapeutic actions for attention-deficit/hyperactivity disorder. Biol Psychiatry 2005; 57: 1410-1415.

15. Riggs PD, Leon SL, Mikulich SK, Pottle LC. An open trial of bupropion for ADHD in adolescents with substance use disorders and conduct disorder. J Am Acad Child Adolesc Psychiatry 1998; 37: 1271-1278.

16. Solhkhah R, Wilens TE, Daly J, Prince JB, Van Patten SL, Biederman J. Bupropion SR for the treatment of substanceabusing outpatient adolescents with attention-deficit/hyperactivity disorder and mood disorders. J Child Adolesc Psychopharmacol 2005; 15: 777-786.

17. Riggs PD, Hall SK, Mikulich-Gilbertson SK, Lohman M, Kayser A. A randomized controlled trial of pemoline for attention-deficit/hyperactivity disorder in substance-abusing adolescents. J Am Acad Child Adolesc Psychiatry 2004; 43: $420-429$.

18. Schubiner $\mathrm{H}$, Saules KK, Arfken $\mathrm{CL}$, Johanson $\mathrm{CE}$, Schuster $\mathrm{CR}$, Lockhart N, et al. Double-blind placebo-controlled trial of methylphenidate in the treatment of adult ADHD patients with comorbid cocaine dependence. Exp Clin Psychopharmacol 2002; 10: 286-294.

19. Carpentier PJ, de Jong CA, Dijkstra BA, Verbrugge CA, Krabbe PF. A controlled trial of methylphenidate in adults with attention deficit/hyperactivity disorder and substance use disorders. Addiction 2005; 100: 1868-1874.

20. Levin FR, Evans SM, Brooks DJ, Garawi F. Treatment of cocaine dependent treatment seekers with adult ADHD: double-blind comparison of methylphenidate and placebo. Drug Alcohol Depend 2007; 87: 20-29.

21. Levin FR, Evans SM, Brooks DJ, Kalbag AS, Garawi F, Nunes EV. Treatment of methadone-maintained patients with adult ADHD: double-blind comparison of methylphenidate, bupropion and placebo. Drug Alcohol Depend 2006; 81: 137-148.

22. Kuperman S, Perry PJ, Gaffney GR, Lund BC, Bever-Stille $\mathrm{KA}$, Arndt $\mathrm{S}$, et al. Bupropion SR vs methylphenidate vs placebo for attention deficit hyperactivity disorder in adults. Ann Clin Psychiatry 2001; 13: 129-134.

23. Chambers RA, Taylor JR, Potenza MN. Developmental neurocircuitry of motivation in adolescence: a critical period of addiction vulnerability. Am J Psychiatry 2003; 160: 10411052.

24. Stansfield KH, Kirstein CL. Neurochemical effects of cocaine in adolescence compared to adulthood. Brain Res 
Dev Brain Res 2005; 159: 119-125.

25. Szobot CM, Rohde LA, Bukstein O, Molina BS, Martins C, Ruaro $\mathrm{P}$, et al. Is attention-deficit/hyperactivity disorder associated with illicit substance use disorders in male adolescents? A community-based case-control study. Addiction 2007; 102: 1122-1130.

26. Liu F, Muniz R, Minami H, Silva RR. Review and comparison of the long acting methylphenidate preparations. Psychiatr Q 2005; 76: 259-269.

27. Rohde LA, Szobot C, Polanczyk G, Schmitz M, Martins S, Tramontina S. Attention-deficit/hyperactivity disorder in a diverse culture: do research and clinical findings support the notion of a cultural construct for the disorder? Biol Psychiatry 2005; 57: 1436-1441.

28. Amorim P. Mini International Neuropsychiatric Interview (MINI): Validação de entrevista breve para diagnóstico de transtornos mentais. Rev Bras Psiquiatr 2000; 22: 106-115.

29. Shaffer D, Gould MS, Brasic J, Ambrosini P, Fisher P, Bird $\mathrm{H}$, et al. A children's global assessment scale (CGAS). Arch Gen Psychiatry 1983; 40: 1228-1231.

30. Associação Brasileira de Empresas de Pesquisas. Critério de Classificação Econômica Brasil. http://www.anep.org.br/ codigosguias/CCEP.pdf. Accessed April 18, 2005.

31. Wechsler D. WISC-III/Manual. New York: The Psychological Corporation; 1991.

32. Wechsler D. Manual of the Wechsler Adult Intelligence
Scale. 3rd edn. San Antonio: The Psychological Corporation; 1997.

33. Swanson J. School-based assessments and Interventions for ADD students. Irvine: KC Publishing; 1992.

34. National Institute of Mental Health. CGI (Clinical Global Impression) scale. Psychopharmacol Bull 1985; 21: 839843.

35. Barkley RA. Attention Deficit Hyperactivity Disorder: A handbook for diagnosis and treatment. New York: Guilford Press; 1990.

36. Gueorguieva R, Krystal JH. Move over ANOVA: progress in analyzing repeated-measures data and its reflection in papers published in the Archives of General Psychiatry. Arch Gen Psychiatry 2004; 61: 310-317.

37. Littell RC, Pendergast J, Natarajan R. Modelling covariance structure in the analysis of repeated measures data. Stat Med 2000; 19: 1793-1819.

38. Koob GF, Le Moal M. Drug abuse: hedonic homeostatic dysregulation. Science 1997; 278: 52-58.

39. Volkow ND, Wang GJ, Fowler JS, Telang F, Maynard L, Logan J, et al. Evidence that methylphenidate enhances the saliency of a mathematical task by increasing dopamine in the human brain. Am J Psychiatry 2004; 161: 1173-1180.

40. Toga AW, Thompson PM, Sowell ER. Mapping brain maturation. Trends Neurosci 2006; 29: 148-159. 